\title{
Malacofauna y Carcinofauna asociada a Rhizophora mangle $(L, 1753)$ en un estuario: río Ranchería, La Guajira, Colombia
}

\section{Malacofauna and Carcinofauna associated with Rhizophora mangle $(\mathrm{L}, 1753)$ in an estuary: Rancheria River, La Guajira, Colombia}

\author{
Geomar Molina-Bolívar ${ }^{1}$ \\ Universidad de La Guajira, Colombia
}

\begin{abstract}
Resumen. Se estudiaron atributos de la malacofauna y carcinofauna asociados a las raíces sumergidas de Rhizophora mangle y su relación con factores fisicoquímicos en el estuario del río Ranchería (La Guajira). Los muestreos se realizaron entre septiembre (2014) y marzo (2015) en cinco sitios; Desembocadura (DB), El Mirador (EM), Villa Fátima (VF), El Pasito (EP) y Valle de Los Cangrejos (VC). Las especies se colectaron cada 15 días en cada muestreo y lugar. Se cortaron al azar dos raíces sumergidas, para así obtener los organismos, los cuales fueron fijados en formalina al $10 \%$ y preservados en alcohol al 70\%. Igualmente se determinaron algunas variables fisicoquímicas del agua, como oxígeno disuelto, salinidad, temperatura y nutrientes (amonio, nitrato y fosforo total). Una vez cuantificados e identificados los organismos se determinó: Abundancia absoluta ( $\mathrm{Ab}$ ), riqueza específica (Margalef - R1) y diversidad (Shannon-Wiener). En total se colectaron 1513 individuos, de los cuales 398 fueron moluscos, distribuidos en las clases bivalva y gasterópoda. La familia Mytilidae fue más representativa y la especie más abundante Mytiliopsis leucophaeta (298 inds.). Se colectó un total de 1115 organismos del subfilum crustacea, de las cuales las familias Palaemoenidae y Aoridae presentaron el mayor número de especies. La especie más abundante fue Americorophium brevis (712 inds.). La principal riqueza especifica $(\mathrm{R} 1=3,23)$ y diversidad $\left(\mathrm{H}^{\prime}=2,12\right)$ se dio en $\mathrm{VF}$, mientras que los menores valores de estas variables se presentaron en $\mathrm{EM}\left(\mathrm{R} 1=0,44 ; \mathrm{H}^{\prime}=0,37\right)$. Se concluyó que, aunque la composición de la comunidad se muestra relativamente diferente a nivel espacial durante el periodo estudiado, no se encontró una relación estadísticamente significativa de esta condición biológica con respecto a los factores fisicoquímicos estudiados.
\end{abstract}

Palabras clave. Malacofauna, carcinofauna, raíces sumergidas, Rhizophora mangle, río Ranchería, La Guajira

\begin{abstract}
We studied malacofaunal and carcinofaunal attributes associated with the submerged roots of Rhizophora mangle and the relationship with physical and chemical factors in the estuary of the Rancheria River (The Guajira). The samplings were carried out between September (2014) and March (2015) in five sampled sites: Desembocadura (DB), El Mirador (EM), Villa Fatima (VF), El Pasito (EP) and Valle de los Cangrejos (VC). The species were collected every 15 days in each sampling location. Two submerged roots were cut at random to obtain the organisms, which were fixed in $10 \%$ formaldehyde and preserved in $70 \%$ alcohol. Also some physicochemical variables of water were determined, such as dissolved oxygen, salinity, temperature, and nutrients (ammonia, nitrate and total phosphorus). Once organisms were quantified and identified, Absolute abundance (Ab), species richness (Margalef - R1) and diversity (Shannon-Wiener) were determined. A total of 1513 individuals were collected, of which 398 individuals were molluscs, distributed in the Bivalve and Gastropoda classes. The Mytilidae family was most representative and the Mytiliopsis leucophaeta was the most abundant species (298 inds). A total of 1115 organisms of the subfilum crustacea were collected of which the Aoridae and Palaemoenidae families had the largest number of species. The most abundant species was Americorophium brevis (712 inds). The highest specific richness $(\mathrm{R} 1=3.23)$ and diversity $\left(\mathrm{H}^{\prime}=2.12\right)$ occurred in VF, whereas the lowest values of these variables were found in EM (R1 =0.44, $\left.H^{\prime}=0.37\right)$. It was concluded that, although the composition of the community was relatively different at the spatial level during the period studied, a statistically significant relation of this biological condition with respect to the physicochemical factors studied was not found.
\end{abstract}

Keywords. Malacofauna, carcinofauna, submerged roots, Rhizophora mangle, Ranchería River, La Guajira 


\section{Introducción}

Los manglares son ecosistemas marino-costeros muy productivos que exportan materia orgánica, caracterizados por adaptarse a condiciones de salinidad e inundación (Barreto, 2001). Desarrollan particularidades como tolerancia a la anoxia, por lo que generalmente se establecen en zonas protegidas y fangosas cercanas a las costas o de orillas de los ríos; las cuales cuentan con ingreso periódico de agua marina o dulce (Nielsen y Quesada, 2006). Al ser ecosistemas altamente productivos, son capaces de soportar y albergar una comunidad de gran importancia ecológica, la cual es principalmente dependiente del mangle como productor primario y base de la cadena trófica (Lema y Polanía, 2007).

Según Valle (2007), el bosque de manglar forma asociaciones en los bordes de las costas tropicales y subtropicales. Los mangles son plantas halofíticas, de las cuales se han idientificado 12 géneros y 8 familias con características marcadas hacia la tolerancia de aguas salobres. A través de sus raíces se adaptan a sustratos inestables lodosos, fangosos, areno-arcillosos y rocosos con bajos contenidos de oxígeno. Dichas estructuras del mangle rojo (Rhizophora mangle Linnaeus, 1753) forman el sustrato para numerosas comunidades con una gran complejidad. Además, son nichos ecológicos verticales estables circundados por sedimentos fangosos o arenosos (Prüsmann y Palacio, 2008; Acosta, Betancourt y Prieto, 2014).

Colombia cuenta con unas 380.000 ha de manglar, de las cuales 292.000 ha están en el Pacífico y 87.000 ha en el Caribe. En La Guajira, se totalizaron 3.131 ha (INVEMAR, 2005). En esta región geográfica (Castellanos y Carabalí, 2015) reportan R. mangle, Avicennia germinans Linnaeus, 1753, Laguncularia racemosa Gaetner, 1805, Conocarpus erecta Linnaeus, 1753 y Pelliciera rhizophorae Triana y Planchon, 1862. Para el Caribe y Centroamérica hay numerosos estudios que hacen referencia a la diversidad biológica asociada a manglares entre estos se destacan los trabajos de Hernández-Alcantará y Solís-Weiss (1994), Prieto, Graterol y Campos (1999), Márquez y Jiménez (2002), Márquez, Blanco-Rambla, Jiménez y Allen (2006), Quiceno y Palacio (2008), Cedeño, Jiménez, Pereda y Allen (2010), Cortés (2010), Guerra-Castro, Cruz-Motta y Conde (2011), Quintana (2011), Hernández-Ávila, Tagliafico y Rago (2013), Ortíz y Blanco (2012), Acosta y colaboradores (2014), Ruíz y Lopez-Portillo (2014). En el Caribe colombiano destacan los trabajos de Reyes (1992), Valle (2007), García y Palacio (2008), Prüsmann y Palacio (2008), Arteta-Bonivento (2009), Cortes (2010), Molina-Bolívar (2010), Ortíz y Blanco (2012), así como tambien Quirós y Arias (2013). El manglar asociado al estuario del río Ranchería presenta diferentes sustratos para el desarrollo de distintas comunidades y son escasos los estudios que involucran variables fisicoquímicas del agua y nutrientes como factores que regulan los atributos de la biodiversidad, especialmente los que se asocian a las raíces de $\mathrm{R}$. mangle.

Es importante realizar un monitoreo constante en los sistemas acuáticos, ya que son el destino final de los contaminantes generados en zonas rurales y urbanas; particularmente, el estuario del río Ranchería se encuentra asociado al poblado de Riohacha, el cual desemboca sus aguas servidas en este ecosistema (Lema y Polanía, 2007). Lo anterior conlleva a la necesidad de conocer mejor las condiciones físico-naturales que lo caracterizan para poder realizar un mejor manejo de los recursos o que permitan la toma de decisiones en pro de la conservación de tal espacio natural en particular. Para esta tarea, uno de los planteamientos iniciales es la necesidad de conocer la diversidad biológica asociada al estuario, a nivel de todos sus microambientes asociados; al partir de esta premisa, los estudios deben enfocarse en la caracterización de los componentes bióticos y abióticos que definen dicho ecosistema estuarino. 
Por lo anterior, se planteó estudiar la relación de la Malacofauna y Carcinofauna asociada a raíces sumergidas de Rhizophora mangle, con algunas variables físicas y químicas en el estuario del río Ranchería, en La Guajira Colombiana, con el propósito de continuar el esfuerzo de caracterizar biológicamente este importante ecosistema para esta región en especial.

\section{Metodología}

El área de estudio se localiza entre $11^{\circ} 33^{\prime} y$ $72^{\circ} 54^{\prime}$ Norte y $11^{\circ} 33$ y $72^{\circ} 53^{\prime}$ Oeste, inmersa en la zona deltaica-estuarina del río Ranchería. Al norte limita con el Mar Caribe y el brazo del Valle de Los Cangrejos, por el sur con el resguardo indígena de la media Guajira, al este con la ciudad de Riohacha y al oeste con la laguna Buenavista (figura 1). En cada sitio de muestreo se realizaron diez (10) muestreos, con una frecuencia aproximada de cada 15 días, entre septiembre (2014) y marzo (2015). Se seleccionaron cinco estaciones o sitios de estudio: Desembocadura (DB), El Mirador (EM), Villa Fátima (VF), El Pasito $(E P)$ y Valle de Los Cangrejos (VC); en cada muestreo, se cortaron a nivel de la línea de marea alta y al azar, dos raíces de mangle sumergidas y sin contacto con el fondo. Estas fueron introducidas en bolsas Ziploc previamente etiquetadas, cuando aún se encontraban sumergidas y antes de realizar el corte. Luego se fijaron en formalina al 10\%, y una vez extraídos los organismos se preservaron en alcohol al 70\%, según la metodología utilizada por Cedeño y colaboradores (2010).

En los laboratorios de Ciencias Biológicas de la Universidad de La Guajira, se separaron los organismos colectados por grupos en un recipiente plástico. Con un estereoscopio (Lexed 40), los organismos fueron cuantificados e identificados hasta el nivel taxonómico más bajo posible con la ayuda de las claves de Díaz y Puyana (1994), Warkme y Tucker-Abbot (1997), Severeyn y Rodríguez (2000), Bitter (2003) y Roldán (1996,
2003). Los ejemplares se conservan en la colección biológica de la Universidad de La Guajira.

Siguiendo las metodologías de UNESCO (1980), se midió in situ la salinidad (UPS) y la temperatura del agua $\left({ }^{\circ} \mathrm{C}\right)$ con un conductímetro (315i WTW 121000 11). Según APHA (1999) y el Ministerio de Salud (1984), se determinó el oxígeno disuelto $(\mathrm{mg} / \mathrm{L})$ con un oxímetro (315i WTW 2B70 0011).

Durante las mediciones in situ, se tomaron muestras de agua superficial $(5-10 \mathrm{~cm}$ de profundidad) en botellas de vidrio de $1 \mathrm{~L}$ de color ámbar, previamente esterilizadas, rotuladas y forradas con papel aluminio. Estas luego se refrigeraron en una nevera de icopor $\left(\sim 6^{\circ} \mathrm{C}\right)$. En los laboratorios del Instituto de Estudios y Aprovechamiento del Agua (INESAG) de la Universidad de La Guajira se determinaron las concentraciones de nutrientes: amonio $\left(\mu \mathrm{mgL}^{-1}\right)$, nitrato $\left(\mu \mathrm{mgL}^{-1}\right)$ y fósforo total $\left(\mu \mathrm{mgL}^{-1}\right)$; siguiendo la metodología establecida por APHA (1999) y el Ministerio de Salud (1984).

Se determinó la Abundancia absoluta $(\mathrm{Ab})$, riqueza específica (Margalef) y diversidad (Shannon-Wiener) con el software PAST ver. 3.01. Inicialmente, se utilizó la estadística descriptiva (promedio, desviación estándar, máximo y mínimo). Posteriormente, para determinar si existen diferencias significativas entre los sitios de estudio se realizó un Análisis de Varianza (ANOVA) de un factor, previa contrastación con pruebas de normalidad y homogeneidad de varianza a las variables biológicas y fisicoquímicas del agua. En aquellos resultados que presentaron diferencias estadísticamente significativas, se aplicó la prueba a posteriori de Duncan (Steel y Torrie, 1960; Sokal y Rohlf, 1979) para establecer dónde se encuentran dichas diferencias. Para observar la relación entre las variables fisicoquímicas y ecológicas, se aplicó un análisis de Correlación de rangos de Spearman (rs) con el software IBM SPSS 22. 


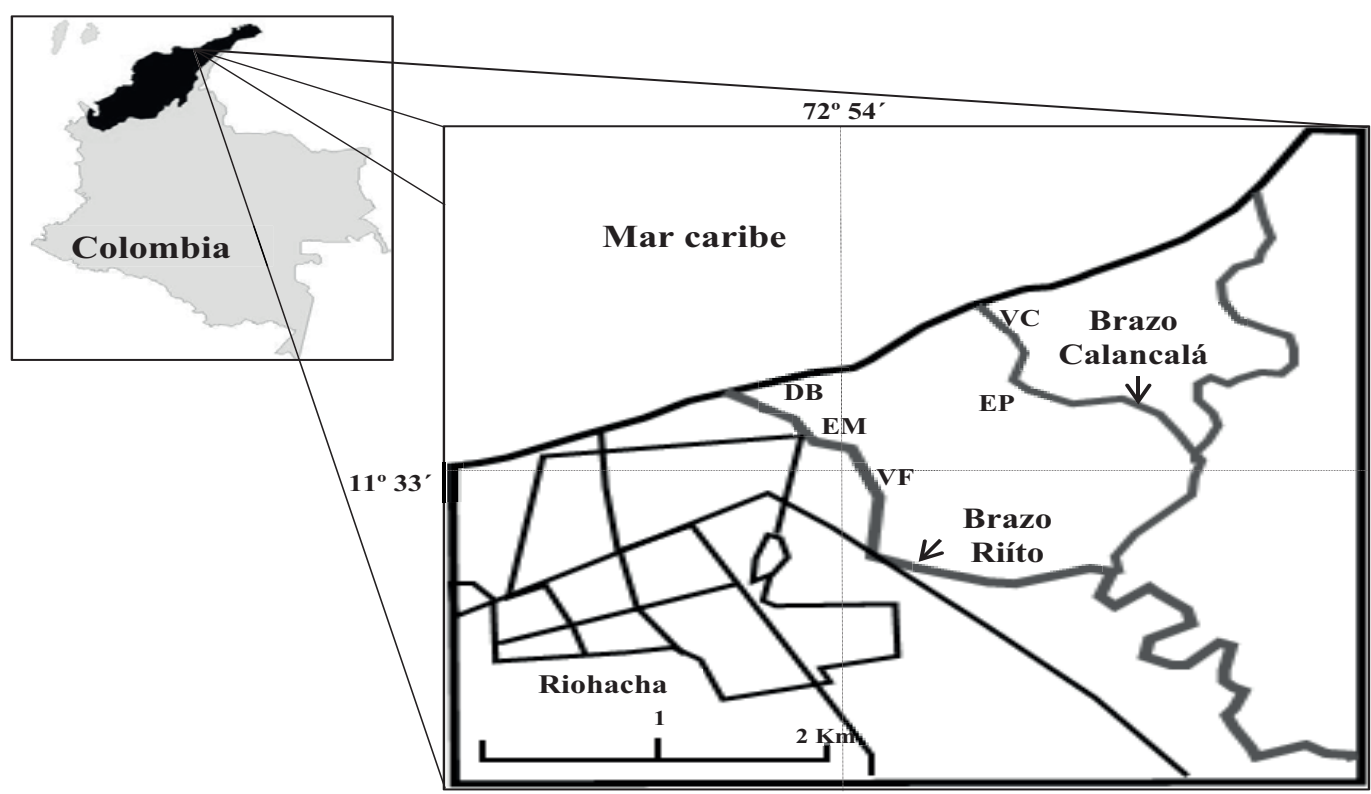

Figura 1. Área de estudio; ubicación de los sitios de muestreos. DB: Desembocadura; EM: El mirador; VF: Villa Fatima; EP: El Pasito; VC: Valle de Los Cangrejos. Nota: Elaboración propia, 2017.

\section{Resultados}

Biológicos: se procesaron 100 raíces de $\mathrm{R}$. mangle y la Abundancia absoluta fue de 1513 individuos pertenecientes a 12 taxones. Los moluscos (398 inds.) estuvieron representados por las clases Bivalvas y Gasterópoda. Por su parte, los artrópodos del subphilum Crustáceo (1115 inds.) estuvieron representados por las Clases Malacostrácea, Maxillopoda y Ostrácoda. Las especies más abundantes fueron los anfípodos Americorophium brevis Shoemaker, 1949 y Grandidierella sp. Coutière, 1904, seguidos por el bivalvo Mytilopsis leucophaeta Conrad, 1831 (tabla 1), por lo que representan estas tres especies el 83,34\% de la abundancia total.

La principal abundancia general se obtuvo en septiembre (381 inds.). Se destaca que no se observaron diferencias estadísticamente significativas para la Abundancia absoluta entre sitios de muestreo ( $\mathrm{F}=0860, \rho=0,495)$. En la tabla 2 , se pueden observar los valores de abundancia por estación y mes de muestreo.

El índice de riqueza especifica de Margalef (R1) presentó valores entre 0,44 y 3,23; el mayor valor en septiembre $(3,23)$ en Villa Fátima y el menor también en septiembre $(0,44)$ en El Mirador. El promedio fue 1,68 $\pm 0,55$. El análisis estadístico mostró que los datos no presentaron diferencias significativas entre las estaciones $(\mathrm{F}=1,604 ; \rho=0,190)$. Para el índice de diversidad de Shannon-Weiner $\left(\mathrm{H}^{\prime}\right)$, los datos oscilaron entre 0,37 y 2,12. El mayor número del índice se dio en septiembre $(2,12)$ en Villa Fátima, y el menor en febrero $(0,37)$ en El Mirador, con un promedio de 1,35 $\pm 0,40$. El análisis estadístico determinó que no se presentaron diferencias significativas entre los sitios $(\mathrm{F}=1,99 ; \rho=0,369)$.

Según la presencia y ausencia de las especies en las estaciones muestreadas, el dendrograma de similaridad de Bray-Curtis presenta tres gru- 


\section{Malacofauna y Carcinofauna asociada a Rhizophora mangle}

Tabla 1

Clase, Familia y Especies de moluscos y crustáceos colectados durante todo el periodo de estudio en las raíces de Rhizophora mangle en el estuario del río Ranchería, La Guajira

\begin{tabular}{|c|c|c|c|c|}
\hline Phylum o Subphylum & Clase & Familia & Especies & Abundancia Total (Inds) \\
\hline \multirow{5}{*}{$\begin{array}{l}\stackrel{\Xi}{0} \\
\stackrel{0}{\Xi} \\
\overline{\overline{0}} \\
\sum\end{array}$} & Bivalvia & Drensenidae & Mytiliopsis leucophaeta & 298 \\
\hline & \multirow{4}{*}{ Gasterópoda } & \multirow{2}{*}{ Neritidae } & Neritina meleagris & 49 \\
\hline & & & Neritina reclivata & 21 \\
\hline & & Hydrobiidae & $\begin{array}{l}\text { Pyrgophorus } \\
\text { platyrachis }\end{array}$ & 18 \\
\hline & & Planorboide & Biomphallaria glabrata & 24 \\
\hline \multirow{7}{*}{ 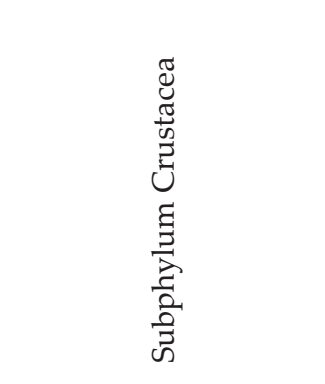 } & \multirow{5}{*}{ Malacostrácea } & Corophiidae & Americorophium brevis & 712 \\
\hline & & Aoriidea & Grandidierella sp. & 251 \\
\hline & & Portunidae & Callinectes sp. & 19 \\
\hline & & Peneaidea & Penaeus sp. & 65 \\
\hline & & Palaemonidae & Periclimenes pauper & 17 \\
\hline & Maxillopoda & Hemicyclopinidae & Cyclopinodes sp. & 17 \\
\hline & Ostrácoda & - & Ostrácoda sp. & 32 \\
\hline
\end{tabular}

Nota. Elaboración propia, 2017

Tabla 2

Abundancias totales de la Malacofauna y Carcinofauna en raíces de R. mangle por estación y mes de muestreo en el estuario del río Ranchería. DB: Desembocadura; EM: El Mirador; VF: Villa Fátima; EP: El Pasito; VC: Valle de Los Cangrejos

\begin{tabular}{ccccccc}
\hline & DB & EM & VF & CG & VF & Total \\
\hline sep_14 & 98 & 76 & 75 & 56 & 76 & 381 \\
oct_14 & 47 & 36 & 25 & 45 & 47 & 200 \\
nov_14 & 31 & 35 & 25 & 30 & 27 & 148 \\
dic_14 & 34 & 56 & 35 & 25 & 34 & 184 \\
ene_15 & 27 & 26 & 55 & 50 & 59 & 217 \\
feb_15 & 23 & 51 & 23 & 43 & 43 & 183 \\
mar_15 & 48 & 20 & 33 & 32 & 67 & 200 \\
Total & 308 & 300 & 271 & 281 & 353 & 1513 \\
\hline
\end{tabular}

Nota. Elaboración propia, 2017 
pos; el primer grupo lo constituye la localidad Desembocadura (DB), el segundo grupo lo forman Valle de Los Cangrejos (VC) y Villa Fátima (VF), y el último grupo se forma con El Mirador (EM) y El Pasito (EP). Entre estos últimos sitios de muestreo hay más similitud ( 70\%) (figura 2).

Fisicoquímicos y nutrientes: la temperatura del agua presentó valores que oscilaron entre 15,9 y $30,7^{\circ} \mathrm{C}$. El valor más alto se presentó en enero $\left(30,7^{\circ} \mathrm{C}\right)$ en Desembocadura y el más bajo en diciembre $\left(15,9^{\circ} \mathrm{C}\right)$ en Villa Fátima. Esta variable no mostró diferencias estadísticamente significativas entre estaciones de muestreo $(\mathrm{F}=0,730$, $\rho=0,576)$ pues presentó un promedio de 26,65 $\pm 2,86^{\circ} \mathrm{C}$. Los registros del oxígeno disuelto fluctuaron entre 0,27 y $6,58 \mathrm{mgL}^{-1}$; los valores más altos y bajos se dieron en septiembre en El Pasito $\left(6,58 \mathrm{mgL}^{-1}\right)$ y El Mirador $\left(0,27 \mathrm{mgL}^{-1}\right)$ respectivamente. No se registró diferencias significativas entre los sitios de muestreo ( $\mathrm{F}=0,734, \rho=0,573)$; el promedio de oxígeno disuelto fue de $3,73 \pm$ $1,97 \mathrm{mgL}^{-1}$. La salinidad registró variaciones entre 0,10 y 42,50 UPS; sin embargo, las localidades no presentaron diferencias significativas $(\mathrm{F}=1,594, \rho=0,192)$, con un valor medio de $14,61 \pm$
10,58 UPS. Dicha variable mostró básicamente una diferencia temporal: hubo un aumento gradual durante el periodo de estudio.

Los registros de amonio (NH4), más alto y bajo se dieron en la localidad El Mirador durante febrero $\left(0,45 \mu \mathrm{molL}^{-1}\right)$ y diciembre $\left(0,00 \mu \mathrm{gL}^{-1}\right)$ respectivamente. Para las estaciones de muestreo no se presentaron diferencias significativas $(\mathrm{F}=1,793, \rho=0,147)$, con un promedio de $0,091 \pm$ $0,15 \mu \mathrm{gL}^{-1}$. El nitrato (NO3) oscilo entre 0,0002 y $0,66{\mu \mathrm{gL}^{-1}}^{-1}$. En El Pasito se registraron los valores más elevados $\left(0,66 \mu \mathrm{mol}-\mathrm{L}^{-1}\right)$ en septiembre. En relación con las estaciones, no se presentaron diferencias significativas $(\mathrm{F}=1,528 ; \rho=0,210)$.

Las concentraciones de fosfato total (PO4) oscilaron entre 0,0068 y $0,77 \mu \mathrm{gL}^{-1}$; el mayor valor se dio en febrero $\left(0,77 \mu \mathrm{gL}^{-1}\right)$ en El Pasito y el más bajo en septiembre $\left(0,0068 \mu \mathrm{gL}^{-1}\right)$ en Villa Fátima. Esta variable en particular mostró diferencias significativas $(\mathrm{F}=5,795, \rho=0,001)$ entre las estaciones. Los sitios Desembocadura, El Mirador y Valle de Los Cangrejos resultaron diferentes de Villa Fátima y El Pasito, ya que se mostró una dinámica diferente de esta variable entre las estaciones estudiadas.

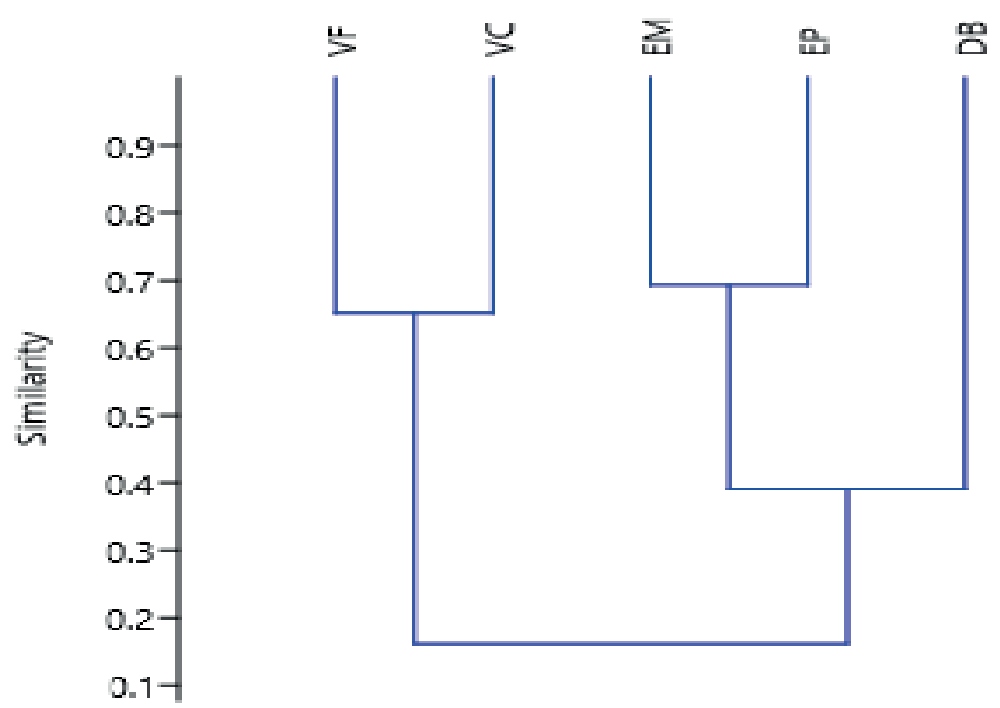

Figura 2. Dendrograma de similaridad de Bray-Curtis (presencia y ausencia de especies) entre las estaciones de muestreo en el estuario del río Ranchería, La Guajira. Elaboración propia, 2017 
Malacofauna y Carcinofauna asociada a Rhizophora mangle

Tabla 3

Valores de las variables fisicoquímicas y nutrientes obtenidos en el estuario del río Ranchería, La Guajira

\begin{tabular}{lcccc}
\hline & Mínimo & Máximo & Promedio & Desviación estándar \\
\hline Temperatura $\left({ }^{\circ} \mathrm{C}\right)$ & 15,90 & 30,7 & 26,65 & 2,87 \\
Oxígeno disuelto $\left(\mathrm{mgL}^{-1}\right)$ & 0,27 & 6,58 & 3,73 & 1,97 \\
Salinidad $(\mathrm{UPS})$ & 0,10 & 42,5 & 14,61 & 10,58 \\
Amonio $\left(\mu \mathrm{gL}^{-1}\right)$ & 0,00 & 0,45 & 0,091 & 0,15 \\
Nitrato $\left(\mu \mathrm{gL}^{-1}\right)$ & 0,0002 & 0,66 & 0,058 & 0,12 \\
Fosfato total $\left(\mu \mathrm{gL}^{-1}\right)$ & 0,0068 & 0,77 & 0,23 & 0,19 \\
\hline
\end{tabular}

Nota. Elaboración propia, 2017

Tabla 4

Valores de correlación de Rangos de Spearman ( $r /$ Alfa=0,05), entre variables biológicas y fisicoquímicas en el área de estudio

\begin{tabular}{lcccc}
\hline & Carácter & Abundancia $(\mathrm{Ab})$ & Shannon-Weiner $\left(\mathrm{H}^{\prime}\right)$ & Margalef $\left(\mathrm{R}_{1}\right)$ \\
\hline \multirow{2}{*}{ Temperatura } & $\mathrm{r}$ & 0,126 & 0,052 & $-0,066$ \\
\multirow{2}{*}{ Oxígeno disuelto } & $p$ & 0,382 & 0,718 & 0,651 \\
& $\mathrm{r}$ & $-0,278$ & 0,226 & 0,123 \\
Salinidad & $p$ & 0,051 & 0,114 & 0,394 \\
& $\mathrm{r}$ & $-0,044$ & $-0,200$ & $-0,116$ \\
Amonio & $p$ & 0,763 & 0,164 & 0,422 \\
& $\mathrm{r}$ & $-0,113$ & 0,121 & 0,074 \\
Nitrato & $p$ & 0,436 & 0,401 & 0,612 \\
& $\mathrm{r}$ & 0,267 & $0,291^{*}$ & 0,128 \\
Fosfato total & $p$ & 0,061 & $0,040^{*}$ & 0,375 \\
& $\mathrm{r}$ & $-0,312^{*}$ & $-0,101$ & $-0,034$ \\
\hline
\end{tabular}

$* p<0,05$. Elaboración propia, 2017

Salvo el fosfato total, las variables fisicoquímicas no diferenciaron un sitio de muestreo de otro significativamente, lo cual indica que la variabilidad temporal solapa los valores de los factores abióticos estudiados en todos los sitios estudiados en el estuario del río Ranchería. Así entonces, se observa un amplio ámbito en los máximos y mínimos de estas variables (tabla 3).

\section{Discusión}

Por una parte, la riqueza de moluscos (5 especies) asociados a las raíces de manglares en el estuario del río Ranchería fue menor a la re- portada por Acosta et al. (2014) en la bahía de Mochima (Venezuela), Cedeño et al. (2010) en la laguna Bocaripo (Venezuela), García y Palacio (2008) en las bahías de Turbo y El Uno (Colombia), y Márquez y Jiménez (2002) en el Golfo Santafé (Venezuela); por otra parte, se presenta un reporte similar al realizado por Prüsmann y Palacio (2008) en el Golfo de Morrosquillo (Colombia). En cuanto a los crustáceos, los resultados obtenidos fueron bajos con referencia a los reportados por Márquez et al. (2006) en el Golfo Santafé (Venezuela), Prüsmann y Palacio (2008) en el Golfo de Morrosquillo (Colombia) y Ruiz 
y López-Portillo (2014) en la laguna La Mancha (México). Los reportes que se hicieron en el presente estudio coinciden con los realizados por Arteta-Bonivento (2009) en el Valle de Los Cangrejos (Colombia).

El número total de especies asociadas a las raíces del mangle rojo en el estuario del río Ranchería fue bajo; ese comportamiento está posiblemente relacionado con el bajo número de especies típicas de ambientes con salinidades fluctuantes y amplia variación del oxígeno disuelto; así como también las actividades antrópicas pueden estar determinando el establecimiento y desarrollo de dichos organismos. En general la salinidad afecta a las especies y hace que exista un cambio significativo en las comunidades, debido a que muchas plantas y animales no se adaptan o desarrollan con cambios bruscos de las condiciones ambientales (Guerra-Castro, Cruz-Motta y Conde, 2011; Márquez y Jiménez, 2002).

A propósito del índice de riqueza de Margalef $\left(R_{1}\right)$ en el estuario del río Ranchería se presentaron valores superiores a los reportados por García y Palacio (2008) en las bahías de Turbo y El Uno (Colombia); el índice de Shannon - Wiener $\left(\mathrm{H}^{\prime}\right)$ fue menor que los reportes hechos en el Golfo Santafé (Venezuela) por Acosta et al. (2014) y en la bahía de Mochima en Venezuela (Márquez y Jiménez, 2002; Márquez et al., 2006).

Los registros de la temperatura de este estudio son similares a los obtenidos por Cedeño et al., (2010), Márquez y Jiménez (2002), Márquez et al., (2006), Molina-Bolívar (2010, 2015) y Silva y Acuña-González (2006). Aunque no se encontraron diferencias significativas entre las estaciones, con excepción del fosfato, la variación observada principalmente a nivel temporal pudo ser el resultado de la disminución del efecto de las mareas, ya que el intercambio de flujos de agua está regido por las épocas de lluvias y sequía, que conllevan a la formación de una barra que desconecta el delta con la costa marina. Las diferencias espaciotemporales en los factores fisicoquímicos suelen estar relacionadas con cambios de las condiciones ambientales a nivel local y estacional, y suelen ser muy dependientes del clima de la zona (Cedeño et al., 2010; García \& Palacio, 2008; Márquez y Jiménez, 2002).

Los valores de oxígeno disuelto obtenido fueron similares a los reportados por Acosta et al., (2014) y Molina-Bolívar (2010), pero menores a los de Márquez y Jiménez (2002). Las variaciones de temperatura y oxígeno disuelto a nivel espacial y temporal pueden deberse principalmente a la influencia de la marea, actividad fotosintética e intensidad eólica, ya que estos factores son más intensos en las estaciones más cercanas a la costa, como Desembocadura y Valle de Los Cangrejos. Sin embargo, las fluctuaciones temporales indican un ambiente muy inestable, que mostró valores de salinidad entre 0,10 y 42,50 UPS, lo que confirma en parte lo indicado por otros autores sobre la condición extrema del estuario del río Ranchería (Lema \& Polanía, 2007).

Los registros de salinidad son similares con los obtenidos por Molina-Bolívar (2010) y Ortíz y Blanco (2012). En el estuario del río Ranchería no se observó la existencia de un gradiente espacial entre las estaciones durante el estudio. No obstante, a nivel de cada localidad se registraron algunas variaciones que son producto de la estacionalidad y los altos índices de evaporación (1600-1800 mm anual), espacialmente en el Valle de Los Cangrejos.

La presencia de M. leucophaeta, A. brevis y Grandidierella sp. como especies dominantes en el ecosistema estuarino del río Ranchería indica que estas se adaptan a las características del substrato (raíces de los manglares); así mismo, presentan tolerancia a las fluctuaciones de salinidad principalmente, mostrando una condición fisiológica eurihalina. 
Los valores registrados para los nutrientes en el estuario del río Ranchería fueron bajos con respecto a los reportados por Silva y Acuña-González (2006) y Seisdedo (2006). La presencia de patrones de distribución de concentraciones de nitrato, nitrógeno amoniacal y fosforo total en los estuarios ha posibilitado la detección de posibles indicios de contaminación. Estos iones participan en el mantenimiento de los ecosistemas acuáticos y actúan como nutrientes esenciales para los sistemas bióticos; con ellos se da inicio a la red trófica.

En relación con la correlación de Spearman, los datos son similares a los obtenidos por (Molina-Bolívar, 2010); son resultados que pueden estar describiendo cierta homogeneidad espacio-temporal, entre las estaciones durante el periodo estudiado, sin poder establecer relaciones claras entre los factores abióticos y bióticos, por lo menos de manera estadísticamente significativa, salvo por los nutrientes nitrato y fosforo. Particularmente, este último se halla muy asociado a sistemas eutróficos (INVEMAR, 2005), por lo que así se podría explicar un poco la correlación inversa (negativa) entre este nutriente y la Abundancia absoluta.

\section{Conclusión}

Al comparar el estuario del río Ranchería con otros del Caribe colombiano, se evidencia que presenta condiciones particulares determinadas por su fisicoquímica, principalmente la salinidad.

La diversidad de moluscos y crustáceos en el estuario del río Ranchería es pobre en comparación con otras áreas de manglar en el Caribe colombiano. Puede deberse a las condiciones ambientales tan fluctuantes del estuario estudiado. La composición de la comunidad se muestra relativamente diferente a nivel espacial durante el periodo de estudio; empero, no se encontró una relación estadísti- camente significativa de esta condición biológica con respecto a las variables ambientales.

El molusco Mytiliopsis leucophaeta, así como los crustáceos Americorophium brevis y Grandidierella sp. fueron las especies dominantes, lo que indica que organismos eurihalinos son los principales representantes de la taxocenósis estudiada.

\section{Agradecimientos}

A la Universidad de La Guajira por el financiamiento y el apoyo de este proyecto. De igual manera a los pescadores de Desembocadura, al profesor Santiago Pichón de la comunidad indígena de El Pasito, por el apoyo permanente. Así mismo, a los estudiantes, hoy biólogos, Oscar Aldana y Erika Viloria Daza, quienes nos apoyaron en el trabajo de campo y laboratorio.

\section{Referencias}

Acosta, V., Betancourt, R. y Prieto, A. (2014). Estructura comunitaria de bivalvos y gastrópodos en raíces de del mangle rojo Rhizophora mangle (Rhizophoraceae) en isla Larga, bahía de Mochima, Venezuela. Rev. Biol. Trop., 62(2), 551-565.

APHA. (1999). Standard methods for examination of water and wastewater. 18 th. Eaton: American Public Health Association, American Water Works Association, Water Environment Federation.

Arteta-Bonivento, R. (2009). Cangrejos en el delta del río Ranchería, Riohacha (Colombia). bol. cient.mus.hist.nat., 13(1), 140-152.

Barnes, R. y Ruppert, E. (1997). Zoología de los invertebrados. México: McGraw-Hill.

Barreto, M. (2001). Análisis estructural de los manglares en el refugio de fauna silvestre Cuaré, Estado Falcón, Venezuela. Acta. Biol. Venez., 43-51.

Bitter, R. (2003). Conchas marinas del Estado Falcón (Venezuela); Guía práctica para su colección e identificación. Coro. 
Castellanos, M.y Carabalí, A. (2015). Deltadel río Ranchería. Una mira biofísca y social antes de la represa. Riohacha: Universidad de La Guajira.

Cedeño, J., Jiménez, M., Pereda, L. y Allen, T. (2010). Abundancia y riqueza de moluscos y crustáceos asociados a las raíces sumergidas del mangle rojo (Rhizophora mangle) en la laguna de Bocaripo, Sucre, Venezuela. Rev. Biol. Trop., 58(3), 213-226.

Cortés, D. (2010). Flora y vegetación asociada a un gradiente de salinidad en el sector de la bahía de Cispatá (Córdoba, Colombia). Bogotá: Universidad Nacional de Colombia.

Díaz, J. y Puyana, M. (1994). Moluscos del Caribe colombiano; un catálogo ilustrado. Santafe de Bogotá: Fundación Natura - INVEMAR.

Fuentes, A., Mogollón, A. y Reyes, W. (2010). Efectos de la salinidad sobre el desarrollo de embriones de Cryphiops caementarius (Crustacea: Palaemonidae) incubados in vitro. Rev. peru. biol., 17(2), 215-218.

García, J. M. y Palacio, J. (2008). Macroinvertebrados asociados a las raíces sumergidas del Mangle Rojo (Rhizophora Mangle) en la bahías de Turboy ElUno, Golfo deUraba (Caribe colombiano). Gestión y Ambiente, 11(3), 55-66.

Guerra-Castro, E., Cruz-Motta, J. y Conde, J. (2011). Cuantificación de la diversidad de especies incrustantes a las raíces de Rhizophora Mangle L. en el Parque Nacional La Restinga. Interciencia, 32(12), 923-930.

Hernández-Alcantará, P. y Solis -Weiss, V. (1994). Algunas comunidades macrobénticas asociadas al manglar (Rhizophora mangle) en laguna de Términos, Golfo de México. Rev. Biol. Trop., 43(1-3), 117-129.

Hernández-Ávila, I., Tagliafico, A. y Rago, N. (2013). Composición y estructura de la macrofauna asociada con agregaciones de dos especies de bivalvos en Isla de Cubagua, Ve- nezuela. Rev. Biol. Trop., 61(2), 669-682.

INVEMAR. (2005). Informe del Estado de los Ambientes Marinos y Costeros en Colombia: año 2005. Santa Marta: Invemar.

Lema, L.yPolanía, J.(2007). Estructura y dinámica del manglar del delta del río Ranchería, Caribe Colombiano. Rev.Biol. Trop., 55(1), 11-21.

Márquez , B. y Jiménez, M. (2002). Moluscos asociados a las raíces sumergidas del mangle rojo Rhizophora mangle, en el Golfo de Santa Fe, Estado Sucre, Venezuela. Rev. Biol. Trop., 50(3/4), 1101-1112.

Márquez, B., Blanco-Rambla, J., Jiménez, M. y Allen, T. (2006). Crustáceos asociados a las raíces del mangle rojo Rhizophora mangle (L.) en el Golfo de Santa Fe, estado Sucre, Venezuela. Ciencias, 14(1), 12-27.

Ministerio de Salud. (26 de junio de 1984). Decreto 1594 de 1984. Bogotá, Colombia.

Molina-Bolívar, G. (2010). Macroinvertebrados bentónicos asociados al ecositema estuarino El Riíto (Riohacha, La Guajira, colombiana). Universidad del Zulia (LUZ), Venezuela.

Molina-Bolívar, G. (2015). Distribución de los macroinvertebrados bentónicos en el ecosistema estuarino "El Riíto", Riohacha - La Guajira colombiana, J. Alcocer,. En J. Alcocer, M. Merino-Ibarra, y E. Escobar-Briones, Tendencias de investigación en Limnología tropical: Perspectivas universitarias en Latinoamérica (págs. 269-277). México D.F-: Asociación mexicana de Linmología, UNAM, y Consejo Nacional de Ciencia y Tecnología.

Morillo, G., Jonte, L., Araujo, I., Ángulo, N. y Herrera, L. (2008). Efectos del nitrogeno y cloruros en la dinámica del fitoplancon del Lago de Maracaibo. Interciencia, 35(8), 575-580.

Nielsen, V. y Quesada, M. (2006). Ambientes marinos de Costa Rica. San José (CR): CIMAR, Conservation International y TNC. 
Noriega, C., Muniz, K., Flores-Montes, J., Macedo, S., Araujo, M., Feitosa, F. y Lacerda, S. (2009). Series temporales de variables hidrobiológicas en un estuario tropical (Brasil). Revista de Biología Marina y Oceanografía, 44(1), 93-108.

Ortiz, L. F. y Blanco, J. F. (2012). Distribución de los gasterópodos del manglar, Neritina virginea (Neritidae) y Littoraria angulifera(Littorinidae) en la Ecorregión Darién, Caribe colombiano. Rev. Biol. Trop., 60(1), 219-232.

Prieto, A., Graterol, A. y Campos, I. (1999). Diversidad de moluscos en dos localidades del golfo de Cariaco, estado Sucre, Venezuela. Caracas: Fundación La Salle de Ciencias Naturales.

Prüsmann, J. y Palacio, J. (2008). Colonización de moluscos y crustáceos en raíces de mangle rojo en la laguna costera de la punta norte del Golfo de Morrosquillo. Gestión y Ambiente, 77-86.

Quiceno, P. A. y Palacio, J. A. (2008). Aporte al conocimiento de los macroinvertebrados asociados a las raices del Mangle (Rizophora Mangle) en la cienaga la Boquilla, municipio de San Onofre, Sucre. Gestión y Ambiente, 11(3), 67-78.

Quintana, M. (2011). Caracterización del mangle rojo (Rhizophora mangle) bajo diferentes presiones en dos sitios de Honduras. Zamorano, Honduras: Escuela Agrícola Panamericana.

Quirós, J. y Arias, J. (2013). Taxocenosis de moluscos y crustáceos en raíces de Rhizophora mangle (Rhizophoracea) en la bahía de Cispatá, Córdoba, Colombia. Acta Biológica Colombiana, 18(2), 329-340.

Reyes, R. (1992). Moluscos, anelidos y crsutaceos asociados a Rhizophora mangle Linneus, en la región de Santa Marta, Caribe colombiano. Caldasia, 17(1), 133-148.
Roldán, G. (1996). Guía para el estudio de los macroinvertebrados acuáticos del Departamento de Antioquia.Bogotá: Universidad de Antioquia.

Roldán, G. (2003). Bioindicación de la calidad de agua en Colombia; Uso del método BMWP/Col. Medellín: Universidad de Antioquia.

Ruiz, M. y Lopez-Portillo, J. (2014). Variación espacio-temporal de la comunidad de macroinvertebrados epibiontes en las raíces del mangle rojo Rhizophora mangle (Rhizophoraceae) en la laguna costera de La Mancha, Veracruz, México. Rev. Biol. Trop., 62(4), 1309-1330.

Seisdedo, M. (2006). Variaciones espaciales y temporales en indicadores de la calidad ambiental de las aguas de la bahía de Cienfuegos, Cuba. Rev. Invest. Mar., 2, 159-165.

Severeyn, H., y Rodríguez, G. (2000). La fauna estuarina: Invertebrados. En. Rodríguez G. (Ed.). El Sistema de Maracaibo (pp. 199-224). Caracas, Venezuela: Instituto de Investigaciónes Científicas (IVIC).

Silva, A. M. y Acuña-González, J. (2006). Caracterización físico-química de dos estuarios en la bahía de Golfito, Golfo Dulce, Pacífico de Costa Rica. Rev. Biol. Trop., 54(1), 241-256.

Sokal, R. y Rohlf, F. (1979). Biometry. Barcelona, España: H. Blume Editores.

Steel, R. y Torrie, J. (1960). Principles and Procedures of Statistics. (With special Reference to the Biological Sciences.). New York, Toronto, London; Estados Unidos, Canada, Reino Unido: McGraw-Hill Book Company.

UNESCO. (1980). La escala de salinidades prácticas de 1978 y la ecuación internacional del estado del agua del mar de 1980. París, Francia:Unesco.

Valle, A. (2007). Estructura y algunos apuntes sobre la fauna asociada a las raíces de Rhizophora mangle (L. 1773) en la ciénaga de Cholón, Isla 
Barú, municipio de Cartagena, Caribe colombiano. Bogotá -Santa Marta: Universidad JTL.

Warkme, G. y Tucker-Abbot, R. (1997). Caribbean Seashells. A guide to the marine Mollusks of Puerto Rico and other west Indian Islands, Bermuda and the lower Florida Keys. New York: Dover Publications.

Recibido: 20 de mayo de 2017

Aceptado: 9 de junio de 2017 